\title{
Correction to: Mesoglycan for pain control after open excisional HAEMOrrhoidectomy (MeHAEMO): an observational multicentre study on behalf of the Italian Society of Colorectal Surgery (SICCR)
}

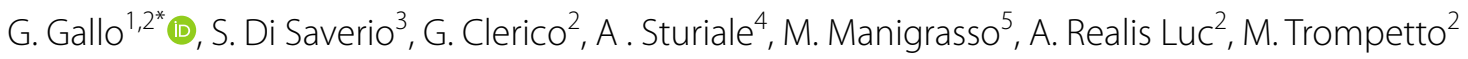
and G. Sammarco ${ }^{6}$ on behalf of the MeHAEMO Working Group

\section{Correction to: BMC Surg (2020) 20:251}

https://doi.org/10.1186/s12893-020-00914-5

Following publication of the original article, we were notified that three of the names of the MeHAEMO working group were incorrectly reported. The correct names are: Marco La Torre, Stefano Mancini and Giovanni De Palma.

The original article has been corrected.

Author details

1 Department of Medical and Surgical Sciences, University of Catanzaro, Catanzaro, Italy. ${ }^{2}$ Department of Colorectal Surgery, S. Rita Clinic, Vercelli, Italy. ${ }^{3}$ Department of General Surgery, University of Insubria, Varese, Italy. ${ }^{4}$ Proctology and Pelvic Floor Clinical Centre, Cisanello University Hospital, Pisa, Italy. ${ }^{5}$ Department of Advanced Biomedical Sciences, Federico II University, Naples, Italy. ${ }^{6}$ Department of Health Sciences, University of Catanzaro, Catanzaro, Italy.

Published online: 11 November 2020

\section{Publisher's Note}

Springer Nature remains neutral with regard to jurisdictional claims in published maps and institutional affiliations. 